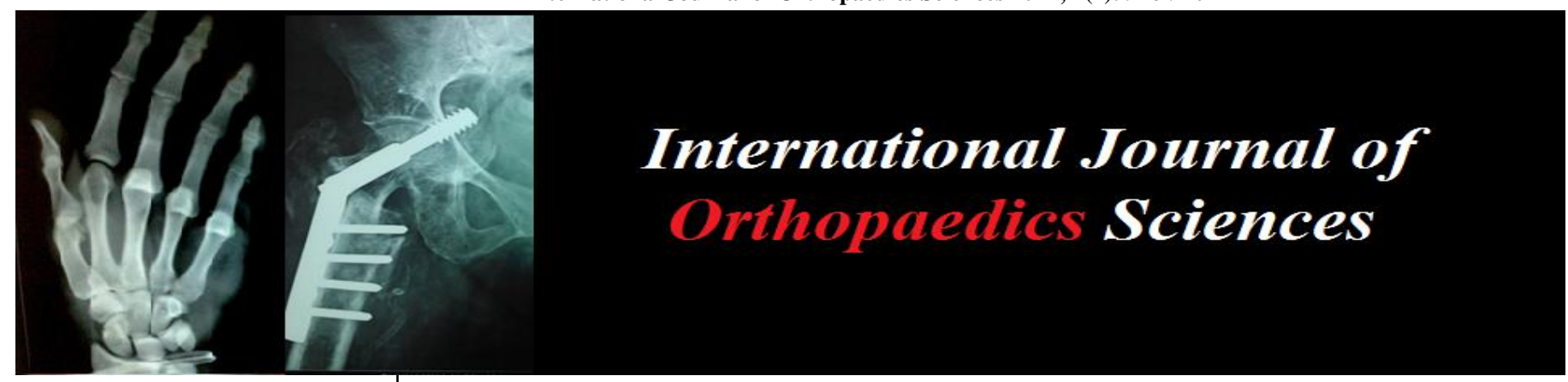

E-ISSN: 2395-1958

P-ISSN: 2706-6630

IJOS 2021; 7(1): 916-920

(C) 2021 IJOS

www.orthopaper.com

Received: 15-11-2020

Accepted: 21-12-2020

\section{Kohli Pavankumar}

Professor, Department of

Orthopaedics, BKL Walawalkar

Rural Medical College and

Hospital, Dervan, Taluka Chiplun,

Ratnagiri, Maharashtra, India

\section{Patel Poorv}

Senior Resident, Department of Orthopaedics, BKL Walawalkar Rural Medical College and

Hospital, Dervan, Taluka Chiplun Ratnagiri, Maharashtra, India

\section{Nadkarni Sunil}

Professor, Department of

Orthopaedics, BKL Walawalkar

Rural Medical College and

Hospital, Dervan, Taluka Chiplun,

Ratnagiri, Maharashtra, India

\section{Sudame Onkar}

Department of Orthopaedics, BKI Walawalkar Rural Medical College and Hospital, Dervan, Taluka Chiplun, Ratnagiri, Maharashtra, India

\section{Dhotkar Kalpit}

Department of Orthopaedics, BKL Walawalkar Rural Medical College and Hospital, Dervan, Taluka

Chiplun, Ratnagiri, Maharashtra, India

Gore Satishchandra

Professor, Department of Orthopaedics, BKL Walawalkar Rural Medical College and Hospital, Dervan, Taluka Chiplun, Ratnagiri, Maharashtra, India

Waybase Hanumant A Arthroplasty Fellow, Department of Orthopaedics, BKL Walawalkar Rural Medical College and Hospital, Dervan, Taluka Chiplun Ratnagiri District - Maharashtra, India

Corresponding Author: Waybase Hanumant $A$ Arthroplasty Fellow, Department of Orthopaedics, BKL Walawalkar Rural Medical College and Hospital, Dervan, Taluka Chiplun, Ratnagiri District - Maharashtra, India

\section{Non traumatic knee effusion: Pointer or Decoy? The commonly missed relationship of knee swelling with Lumbar disc disease: "The Dervan knee effusion sign"}

\author{
Kohli Pavankumar, Patel Poorv, Nadkarni Sunil, Sudame Onkar, \\ Dhotkar Kalpit, Gore Satishchandra and Waybase Hanumant A
}

DOI: https://doi.org/10.22271/ortho.2021.v7.i1n.2588

\section{Abstract}

Introduction: Knee pain and swelling are known amongst orthopedic surgeons as symptoms of a local pathology arising from the knee joint area. It is also often used by patients as an 'umbrella term'. Although in most cases the causative pathology may be the knee joint itself, however, this umbrella term can cause prejudice among clinicians to look at only locally and not think of a lumbar pathology as a cause of knee pain and swelling. The objective of this pilot study was to make clinicians aware that causes knee pain and swelling may be beyond the anatomical confinements of the knee.

Material and Methods: This is a prospective study in which we evaluated consecutive patients with non-traumatic knee joint pain and effusion coming in our hospital from 1st January to 1st February 2020. Detailed history and clinical examination were taken. After ruling out obvious causes of non-traumatic knee pain and effusion in these patients, we included 38 patients based on our inclusion and exclusion criteria. Written informed consent was taken. They were evaluated for knee joint with radiographs and lumbar spine with radiographs and MRI. Arthrocentesis and synovial fluid evaluation were done.

Results: After synovial fluid examination, 2 patients were found to have subclinical inflammatory arthritis and were excluded out of the study. 36 of the 38 patients had a normal study on arthrocentesis. 2 out of these 36 patients had no abnormalities on spine MRI. Out of the remaining 34, 30 had disc degenerative disease at L45 or L5S1 (25 patient L4-5, 3 patient L5-S1, 4 patients with both L4-5 and L5S1), 1 patient had a L34 disc degenerative disease, 3 patients had Grade 2 L45 spondylolisthesis with mild or no back pain.

Conclusion: Although a disc degeneration can be seen incidentally in many asymptomatic patients, based on our observations we think that causes like "autonomic" overload can be one of the unheard reasons of knee pain and effusion. The clinician must be aware of possible causes of knee pain and swelling beyond the anatomical confinements of knee and that lumbar spine pathology need also to be ruled out. The clinician must not let prejudice rule his clinical judgement. We don't claim direct association of lumbar disc degeneration and knee pain and swelling in this study. Further studies are needed.

Keywords: knee effusion, knee Swelling, lumbar disc, degenerative disc

\section{Introduction}

Knee pain with swelling is one of the most common symptom presentations with which a patient comes to an orthopaedic surgeon.It requires a systematic diagnostic approach. However, the knee joint is susceptible to effusions secondary to a wide variety of nontraumatic causes (e.g. inflammatory, septic, sympathetic etc.). Each cause needs to be identified and specific treatment needs to be given. Arthroplasty surgeries are doneroutinely now for osteoarthritis knee; however, arthroplasty surgeons are often faced with dissatisfied patients complaining of lingering knee pain, often behindthe knee ${ }^{[1]}$. This may be due to the fact that sources of knee pain can also be secondary to pain generators in lumbar spine ${ }^{[2-5]}$.

Most of the causes of non-traumatic knee pain and swelling can be diagnosed based on clinical and radiological examination (Xray's and MRI's'). Osteoarthritis being one of the most common causes of knee pain and swelling can be easily diagnosed clinically and radiographically. 
Apart from clinical and radiological evaluation, other causes of non-traumatic knee swelling need arthrocentesis and synovial fluid examination to differentiate between each other.

Sympathetic joint effusion (SJE) is poorly understood clinical entity not yet been well characterised in the literature with its $\mathrm{n}$ annualized incidence of $1.2 \%{ }^{[6]}$. The exact cause of SJE is not known with early theories being proposed as vascular permeability and localized oedema from inflammation or immobilization, imbalance of synovial fluid permeability due to disruption of joint fluid turnover and lymphatic system ${ }^{[7,8]}$. SJE is usually thought to be associated with concurrent adjacent pathology in the same limb, however, most of the times it may not be so.

Chronic regional pain syndrome (CRPS) is another entity whose pathogenesis is still obscureand is clinically characterized by sensory, autonomic and motor disturbances 9 . It is usually seen with extremity trauma, but can also be seen in association with central lesions (e.g. stroke, cervical cord injury). Pathophysiologically, there is evidence of functional changes in central nervous system and for involvement of peripheral inflammatory process ${ }^{[10]}$.

We clinicians are aware that the site of pain is not necessarily the source of pain. Based on our previous experience of concomitant knee pain with lumbar disc degeneration (Dervan Dermatome Syndrome) ${ }^{[1]}$ and the background of theories pathogenesis of SJE ${ }^{[6]}$ and CRPS ${ }^{[10]}$, we think that these cases of unexplained knee pain and effusion can also be due to "autonomicoverload" secondary to nerve root irritation in lumbar disc degenerative disease. Theobjective of this study was to make the clinician aware of that causes of knee pain and swelling may not be limited to anatomical confinements of knee but may also be due to a lumbar spine pathology. The clinician must not let prejudice rule his clinical judgement.

\section{Material and Methods}

This is a prospective observational study in which we evaluated consecutive patients with non-traumatic knee joint pain and effusion coming in BKL Walawalkar Rural Medical College and Research Centre, Dervan, Maharashtra, India from $1^{\text {st }}$ January to $1^{\text {st }}$ February 2020. Detailed history, clinical and radiologically examination was done and patients were selected based on the following criteria:

\section{Inclusion criteria}

1. Non-traumatic knee pain with swelling

2. Age more than 20 years

3. All patients who gave consent for the study

\section{Exclusion criteria}

1. Traumatic knee pain and swelling

2. Post-traumatic arthritis

3. Inflammatory arthritis with or without its systemic associations (e.g. inflammatory bowel disease)

4. Septic arthritis

5. Intra-articular malignancies

6. Neuropathic joints

7. Neurological disorders (e.g. diabetic neuropathies, cerebrovascular stroke)

8. Previous history of knee surgery

9. Sympathetic knee joint effusions with identified pathologies in the vicinity (e.g. osteomyelitis, bone tumours, bursitis around patella, any ipsilateral hip pathology, thigh or leg hematoma)
After selecting the patients based on above criteria they were evaluated withstanding AP and Lateral view radiographs of the affected knee. They further underwent X- raysand MRI of Lumbosacral spine withaxial and sagittal view (T1 and T2 weighted images). Patients with and without lumbar spine abnormalities were noted. All patients were examined clinically for knee pain and swelling,general examination done to rule out any systemic neurological disorder. Patients then underwent arthrocentesis of the affected knee and synovial fluid examination was done which includedaerobic culture, gram and $\mathrm{ZN}$ staining and routine cytology.

\section{Results}

38 patients were included in our study based on our eligibility criteria, of which 2 were excluded based on synovial fluid analysis. All the remaining 36 patients had normal synovial fluid examination with mild (squeeze test) to moderate (patellar tap) effusion (Image 1 and 2). All the 36 patients had symptoms of chronic mechanical low back pain revealed after asking leading questions. All 36 patients presented with some pattern of radicular leg pain corresponding to the side of knee effusion and pain. The average age of patients was 62 (4073 yrs) with mostly male patients (25 males, 11 female). Mostly single knee was involvement with almost equal predilection to each side (Table 1)

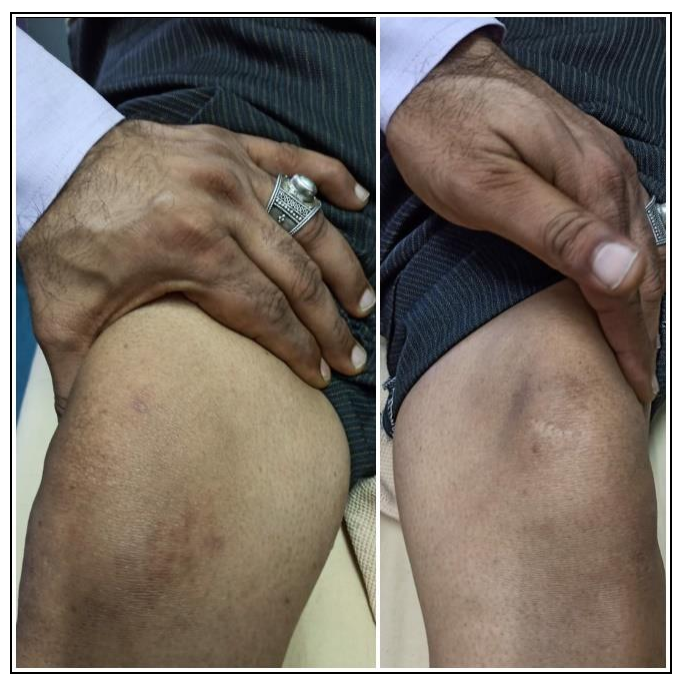

Image 1: 40-year male with mild right knee effusion

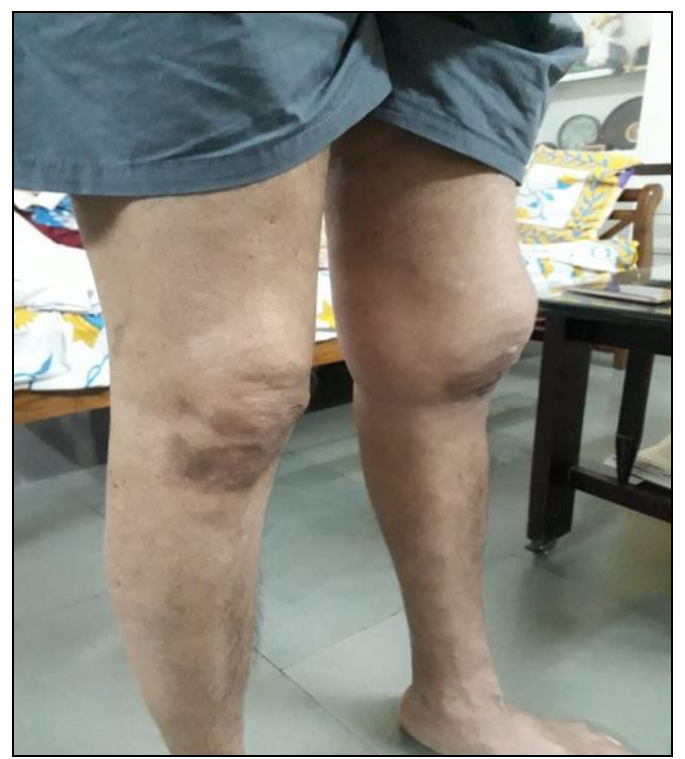




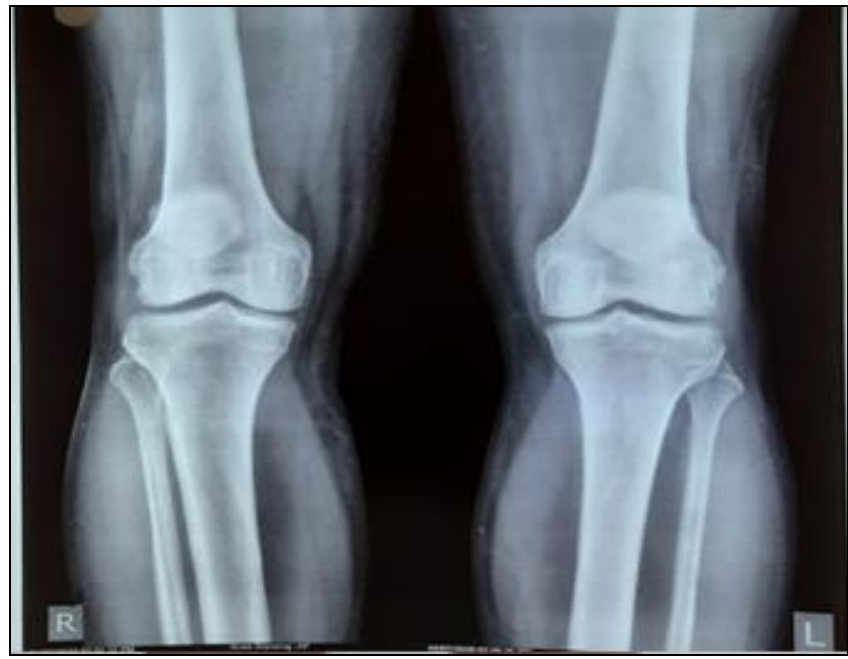

Image 2: 62-year male with bilateral knee moderate effusion with his bilateral knee standing AP Xray's
Table 1: Side of involvement

\begin{tabular}{|c|c|c|}
\hline Side of involvement & No. of patients & Percentage \\
\hline Right side & $16 / 36$ & 44.4 \\
\hline Left side & $17 / 36$ & 47.2 \\
\hline Bilateral & $3 / 36$ & 8.3 \\
\hline
\end{tabular}

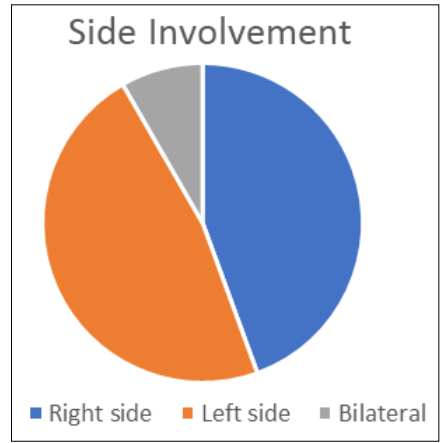

Diagram 1: Side of involvement (in percentage)

Out of the 36 patients, lumbar disc degenerative changes were seen in 34 patients

Table 2: Comparison of patients with knee effusion and pain with or without lumbar disc degeneration (on MRI)

\begin{tabular}{|c|c|c|}
\hline Patients with knee effusion and pain with or without lumbar disc degeneration (on MRI) & No. of patients & Percentage \\
\hline With MRI changes & $34 / 36$ & 94.4 \\
\hline Without MRI changes & $2 / 36$ & 5.6 \\
\hline
\end{tabular}

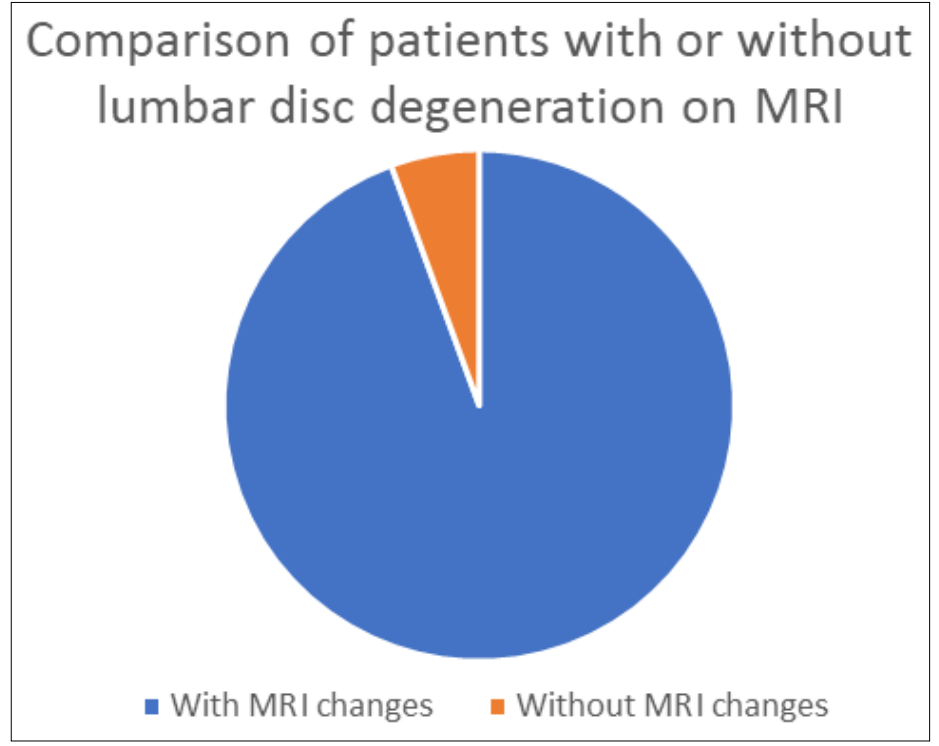

Diagram 2: Comparison of patients with or without lumbar disc degeneration on MRI (in percentage)

Out of the remaining 34, 30 had disc degenerative disease at L45 or L5S1 (25 patient L4-5, 3 patient L5-S1, 4 patients with both L4-5 and L5-S1), 1 patient had a L34 disc degenerative disease, 3 patients had Grade 2 L45 spondylolisthesis with mild or no back pain.

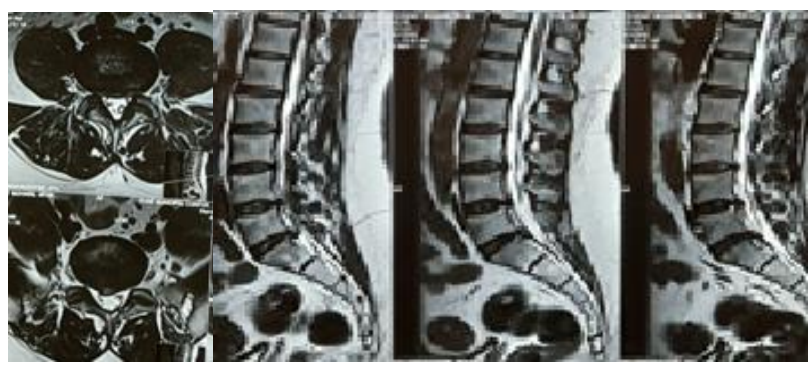

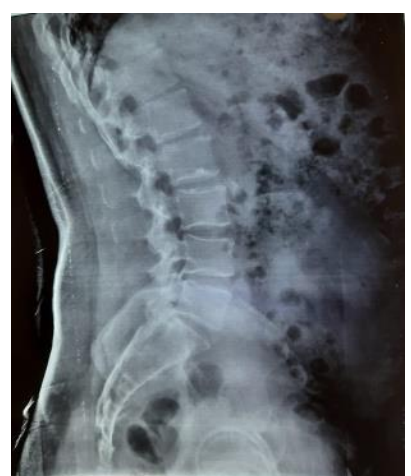

Image 3: MRI Lumbosacral spine (axial and sagittal sections) and LS spine standing lateral xray of a 44-year-old male labourer with L4-5 Levels and L5-S1 disc protrusion (axial sections) 
Table 3: Lumbar spine involvement pattern on MRI and Xray's

\begin{tabular}{|c|c|c|}
\hline Lumbar spine involvement pattern on MRI and Xray's & No. of Patients & Percentage \\
\hline L45 disc involvement & $25 / 36$ & 69.4 \\
\hline L5S1 disc involvement & $3 / 36$ & 8.3 \\
\hline L45 and L5S1 disc involvement & $4 / 36$ & 11.1 \\
\hline L34 disc involvement & $1 / 36$ & 2.8 \\
\hline L45 spondylolisthesis & $3 / 36$ (both Grade 2) & 8.3 \\
\hline
\end{tabular}

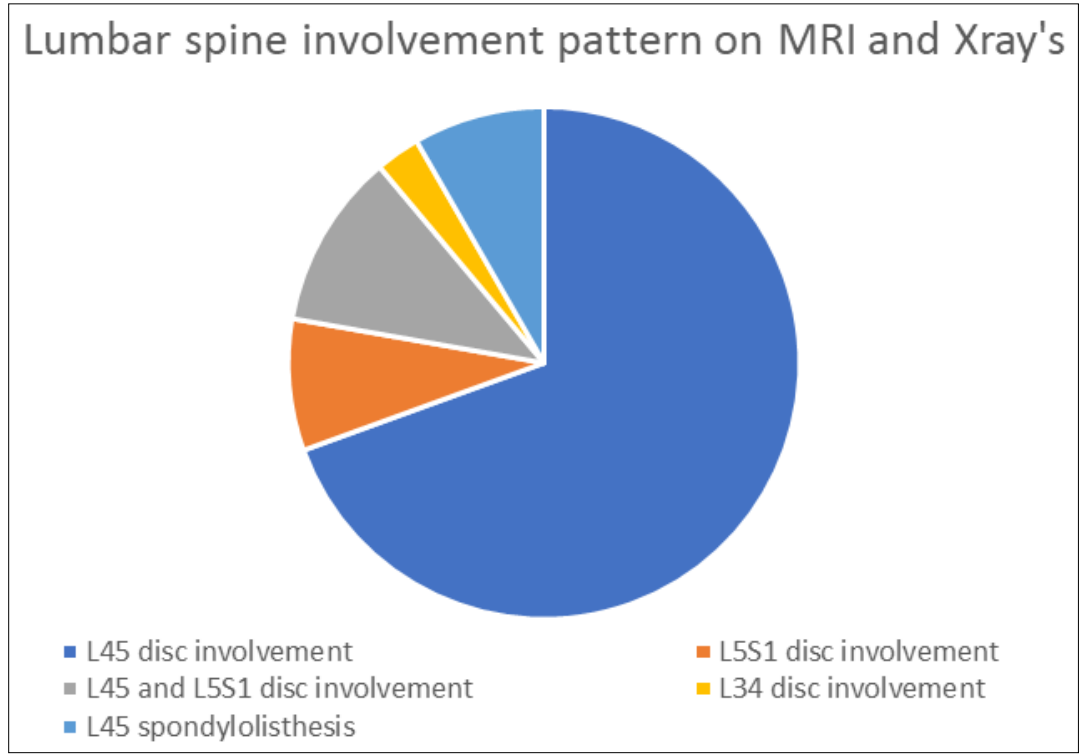

Diagram 3: Lumbar spine involvement pattern on MRI and Xray's (in percentage)

\section{Discussion}

Knee joint sensory innervation is complex with high degrees of anatomical variability. Knee joint capsule is supplied by branches from 4 nerves - femoral, saphenous, obturator, sciatic and its branches (tibial and common peroneal) ${ }^{[11]}$. The root values of femoral nerve (L2, L3, L4), saphenous nerve (L2, L3, L4), obturator nerve (L2, L3, L4) and sciatic (L4-S2) are established in anatomical studies ${ }^{[12]}$. Synovial fluid is an ultrafiltrate of the blood plasma, which is concentrated by its virtue of filtration through synovial membrane ${ }^{[13]}$. Synovium has a rich nerve supply with the sympathetic nerve system around vascular networks ${ }^{[14]}$.

According to the International Association for the Study of Pain (IASP), CRPS is divided into two types. In CRPS type I (reflex sympathetic dystrophy), minor injuries to a limb or lesions in remote body areas precede the onset of symptoms. CRPS type II (causalgia) develops after injury to a major peripheral nerve ${ }^{[15]}$. Pain in or around the injured sites in CRPS may indicate that the local tissue injury might be the cause, however, it fails to explain why would there be autonomic disturbances which have central thermoregulation. Alterations in central nervous system may play a role CRPS. But it is not clear whether these are primary changes or secondary to pain ${ }^{[10]}$. CRPS is a clear example of how the central nervous system can play an important role in the development of systems - "pain generator" being away from the site of injury.

Lower limb radiculopathy was thought to be due to pressure alone on the nerve root by a herniated disc. We now know that chemical factors are as important as the mechanical factors. Nucleus pulposus (NP) induces functional and structural nerve root changes by substances located at the surface of NP cells ${ }^{[16]}$. Radiculopathy in early stages is due to increased excitability and sensitization of DRG and root, resulting in radicular pain along the distribution of the peripheral sensory nerve fibres. Excitability and sensitization of the nerve fibres at that stage may respond well to treatments directed at stabilizing oversensitive nerve membranes - local anaesthetics (Sodium channel blockers) ${ }^{[16]}$. It has been established that giving distal nerve root blocks along the course of affected nerve - L5(deep peroneal nerve) and S1 (sural nerve) ${ }^{[16]}$. Radicular pain again is an example of "pain generator" being away from the region of pain perception.

The exact aetiology and mechanism of SJE is unknown. Theories include changes in vascular permeability and localized oedema from inflammation or immobilization, imbalance of synovial fluid permeability that is due to disruption of joint fluid turnover and lymphatic system. It usually mimics inflammatory or infective arthritis, but can be differentiated by arthrocentesis. In SJE, synovial fluid WBC count is $<2000 / \mathrm{mm}^{3}$. With knee joint being the most commonly involved, it is thought that SJE is usually seen due to an adjacent pathology (e.g osteomyelitis of femur /tibia, trauma, tumour) ${ }^{[8]}$. However, it may not always be the case and the cause may yet be unknown. The retrospective study of Tan et al. ${ }^{[8]}$ of 72 patients is the largest cohort on disease characterization of SJE/SSE to date and the first clinical study dedicated to this topic. In their study, 61 patients $(85 \%)$ had knee joint affection with concurrent adjacent pathology was seen in 21/61 knee joints (34\%) which also included 2 cases of recent hip joint surgeries. In the rest of the 40 knee joints (66\%) no concurrent adjacent pathology was seen nor could the authors give an explanation.

In our study, out of total 38 patients, 2 patients had to be excluded based on synovial fluid analysis. Of the remaining 36 patients, 2 had no abnormalities in spine MRI. 34/36 (94\%) patients had some sort of spine pathology based on Xray's and MRI. The most common levels affected on imaging were L45 and L5S1. Based on anatomical correlation L5 and S1 (traversing roots) would be the most common roots affected except in the case of foraminal affection which none 
of our patients had. According to the traditional teaching, the representative dermatome of the knee joint is L3 and L4. To explain this conundrum, we may refer to our previous discussion that the site of injury (CRPS) and region of pain perception (radiculopathy) may be different from the site of actual pathology. Also, anatomical studies have shown that the knee joint has innervations from $\mathrm{L} 2$ to $\mathrm{S} 2$ roots ${ }^{[11,12]}$, so irritation of any root along with the reach nerve supply of the synovium [13] may be a reason for this presentation. Additionally, based on our observations of the study by Tan et $a l$, we can ponder on the possibility that lumbar spine affections can also be the source of knee joint. All the 36 patients didn't give history of low back pain as a presenting symptom. Only after asking them leading questions did they agree that they also had symptoms of chronic mechanical low back pain. So, we may say that lumbar disc degeneration causing knee pain and effusion can be considered as a diagnosis of exclusion.

Based on this study, we don't claim a direct correlation of knee joint effusion and pain due to nerve root irritation secondary to lumbar disc degeneration. We present a conjecture of "autonomic system overload" as a causative factor and we suggest concomitant lumbar disc degeneration tobe included as one of the differential diagnosis of knee pain and effusion of unknown aetiology as a diagnosis of exclusion. At this stage we would like to propose a "Dervan Knee Effusion Sign" as a sign to confirm the existence of lumbar degenerative spine pathology in a case with a nontraumatic knee pain and effusion. However, we do recommend that further studies are needed to establish this causative factor. We are aware that lumbar spine abnormalities can be seen in asymptomatic individuals as well with varying presentations on MRI, so we had included the all patients above 20 years old ${ }^{[17]}$. The objective of this study was to make the clinician aware that causes of knee pain and swelling may not be limited to anatomical confinements of knee joint and surrounding area but may also be due to a lumbar spine pathology. The clinician must not let prejudice rule his clinical judgement.

\section{Conclusion}

Based on our previous experiences of pain management, current observations and published literature review, we suggest that lumbar spine disc degenerative disease be included as one of the differential diagnosis of knee pain and effusion of unknown aetiology as a diagnosis of exclusion. However, we do recommend that further studies are needed to establish this claim. We think that this might be due "autonomic system overload" of nerve root irritation in the lumbar spine, which may cause knee pain and effusion. The clinician must be aware of possible causes of knee pain and swelling beyond the anatomical confinements of knee and that lumbar spine pathology need also to be ruled out. The clinician must not let prejudice rule his clinical judgement

\section{References}

1. Stirling Bryan, Laurie J, Goldsmith Jennifer C. Davis, etal. Revisiting patient satisfaction following total knee arthroplasty: a longitudinal observational study. BMC Musculoskelet Disord 2018;19:423.

2. Pavankumar Kohli, Ankush Nawale, Sushant Chavan, Satishchandra Gore, Sunil Nadkarni. Dervan dermatome syndrome often missed but surprisingly common coexistent spine pathology in 'Knee Pain', ignore at your own risk. Int J Orthop Sci 2018;4(4):649-653.
3. Mohammad Rahbar et al. Association between knee pain and low back pain. J Pak Med Assoc 2015;65(6):626-31.

4. Wolfe F, Hawley D, Peloso P, Wilson K, Anderson J. Back pain in osteoarthritis of the knee. Arthritis Care Res 1996;9:376-83.

5. Hong AN, Lim MD, Eun-Kyoo Song MD, et al. Causes of Aseptic Persistent Pain after Total Knee Arthroplasty. Clin Orthop Surg 2017;9(1):50-56.

6. Strickland RW, Raskin RJ, Welton RC. Sympathetic synovial effusions associated with septic arthritis and bursitis. Arthritis Rheum 1985;28:941-3.

7. Levick JR, McDonald JN. Fluid movement across synovium in healthy joints: role of synovial fluid macromolecules. Ann Rheum Dis 1995;54:417-423.

8. Tan IJ, Barlow JL. Sympathetic Joint Effusion in an Urban Hospital. ACR Open Rheumatol 2019;1(1):37-42.

9. Christina Misidou, Charalampos Papagoras. Complex Regional Pain Syndrome: An update Mediterr J Rheumatol 2019;30(1):16-25.

10. Wasner G, Schattschneider J, Binder A, Baron R. Complex regional pain syndrome- diagnostic, mechanisms, CNS involvement and therapy. Spinal Cord 2003;41:61-75.

11. Fonkoué L, Behets C, Kouassi JK, Coyette M, Detrembleur C, Thienpont E, et al. Distribution of sensory nerves supplying the knee joint capsule and implications for genicular blockade and radiofrequency ablation: an anatomical study. Surg Radiol Anat 2019;41(12):1461-1471.

12. Devon Rubin. Brachial and lumbosacral plexopathies: A review. Clin Neurophysiol Pract 2020;5:173-193.

13. Tamer TM. Hyaluronan and synovial joint: function, distribution and healing. Interdiscip Toxicol. 2013 Sep;6(3):111-25.

14. Veronica Macchi, Elena Stocco, Carla Stecco, Elisa Belluzzi, Marta Favero, Andrea Porzionato et al. The infrapatellar fat pad and the synovial membrane: an anatomo-functional unit. J Anat. 2018;233(2):146-154

15. Merskey H, Bogduk N. Classification of chronic pain: descriptions of chronic pain syndromes and definition of terms. Seattle: IASP Press, 1995.

16. Satishchandra Gore, Sunil Nadkarni. Sciatica: Detection and Confirmation by New Method. Int J Spine Surg 2014.

17. Brinjikji W, Luetmer PH, Comstock B, Bresnahan BW, Chen LE, Deyo RA, et al. Systematic Literature Review of Imaging Features of Spinal Degeneration in Asymptomatic Populations. AJNR Am J Neuroradiol 2015;36(4):811-816. 